\title{
Composición Factorial de una Escala de Autoeficacia en el Ámbito Sociocultural en Universitarios de Ingeniería
}

\author{
Francisco Muñoz, María C. Zueck, Gabriel Gastélum y Julio C. Guedea \\ Universidad Autónoma de Chihuahua. Facultad de Educación Física y Ciencias del Deporte. DES \\ Salud CAs 101 y 121. Calle Escorza 900, CP 31000 Chihuahua, Chih. México. \\ (e-mail: fmunoz@uach.mx,mzueck@uach.mx,gastelum@uach.mx y jcguedea@uach.mx)
}

Recibido Mar. 02, 2012; Aceptado Abr. 24, 2012; Versión final recibida Abr. 30, 2012

\begin{abstract}
Resumen
El presente estudio analiza las propiedades psicométricas de una escala de autoeficacia en el ámbito sociocultural en universitarios de ingeniería. La muestra total fue de 592 sujetos; 143 mujeres y 449 hombres estudiantes de ingeniería, con una edad media de 18.54 años. La estructura factorial del cuestionario se analizó a través de análisis factoriales exploratorio y confirmatorio. Los análisis, mostraron que una estructura bifactorial es viable y adecuada; con indicadores de ajuste, de fiabilidad y de validez apropiados. Además, los resultados de los análisis factoriales llevados a cabo con las submuestras 1 y 2 , indican la existencia de fuertes evidencias de estabilidad de la estructura factorial, hasta que no se demuestre lo contrario.
\end{abstract}

Palabras clave: psicometría, validez de las pruebas, creencias del estudiante, actitudes del estudiante, pruebas psicológicas

\section{Factor structure of a self-efficacy scale in the sociocultural ambit on university students of engineering}

\begin{abstract}
The present study analyses the psychometric properties of the self-efficacy scale in the sociocultural ambit on university students of engineering. The total sample comprised 592 participants; 143 female and 449 male students of engineering, with a mean age of 18.5 years. The factor structure of the questionnaire was analyzed through two separate exploratory and confirmatory factor analysis. Psychometric analysis showed that a two-factorial structure was viable and adequate; with appropriate indicators of adjustment, reliability and validity. In addition, factor analysis conducted with subsamples 1 and 2 indicated the presence of strong evidence of factor structure stability, until proven otherwise.
\end{abstract}

Keywords: psychometry, validity of tests, student's beliefs, student's attitudes, psychological tests 


\section{INTRODUCCIÓN}

Las creencias que tienen las personas acerca de sí mismas son claves para el control y la competencia personal frente a los problemas, desafíos y decisiones que tienen que ir enfrentando durante su vida. Entre las creencias de autorreferencia que usan los individuos al interactuar con su ambiente están las creencias de autoeficacia. El término autoeficacia se puede definir como los juicios que cada individuo hace sobre sus capacidades, en base a los cuales organizará y ejecutará sus actos de modo que le permitan alcanzar el rendimiento deseado (Bandura, 1997); o como las creencias que tiene la persona sobre sus capacidades para organizar y ejecutar caminos para la acción requeridos en situaciones esperadas o basadas en niveles de rendimiento (Blanco et al., 2011).

La aplicación de la teoría de la autoeficacia de Bandura en el ámbito educativo muestra cómo los estudiantes con altas expectativas de autoeficacia gozan de mayor motivación académica (Brown et al. 2008; Caprara et al. 2008). Asimismo, obtienen mejores resultados, son más capaces de autorregular eficazmente su aprendizaje y muestran mayor motivación intrínseca cuando aprenden (Cartagena, 2008; Salanova et al., 2011). En consecuencia, la mejora de las expectativas de autoeficacia incrementa la motivación y el rendimiento en las tareas de aprendizaje (Adeyemo, 2007). Por tanto, no basta con ser capaz de, es preciso juzgarse capaz. Capaz de utilizar las habilidades y destrezas personales ante circunstancias muy diversas, incluso en las reacciones emocionales que se experimentan ante situaciones difíciles (Blanco et al., 2011).

Como una muestra de la importancia de la autoeficacia en el ámbito académico, podemos decir que esta revela por qué las personas con el mismo nivel de habilidad y conocimiento presentan conductas y/o resultados diferentes, o por qué las personas actúan en disonancia con sus habilidades (Bandura, 1982; Pérez et al., 2011). Lo anterior se explica porque el rendimiento académico adecuado también depende de la eficacia percibida para manejar demandas académicas exitosamente. Por ello, las creencias de autoeficacia en la propia capacidad son imprescindibles para dominar las actividades académicas; ya que los estudiantes que confían en sus capacidades se sienten más motivados para alcanzar sus metas (Blanco, 2010). Así mismo, las personas que dudan de sus capacidades pueden creer que las cosas son más difíciles de lo que realmente son, creencia que genera tensión, depresión y una visión estrecha para resolver problemas (Vera et al., 2011). Se ha evidenciado que un bajo nivel de autoeficacia puede ser responsable no sólo de disminución del rendimiento escolar e interés hacia el estudio, sino también de comportamientos inadaptados en jóvenes (Zimmerman y Kitsantas, 2005), de ahí la importancia de que la educación fortalezca el desarrollo de la competencia académica en el estudiante y fomente habilidades que le permitan creer en sus propias capacidades (Carbonero y Merino, 2008; Ornelas et al., 2011).

Las creencias que tienen las personas sobre sí mismas representan un factor básico para el logro de sus actividades o la toma de decisiones que enfrentarán a lo largo de su vida. Cuanto mayor sea la eficacia percibida, mayor el grado de esfuerzo que se invierta y mayor también la persistencia en el logro de la meta propuesta; situación de suma importancia para que una persona que se encuentra en un proceso de aprendizaje tenga éxito (Bandura, 1997; Ornelas et al., en prensa).

La propia motivación impulsa al ser humano a emprender conductas específicas en función de los logros que éste pretende alcanzar. Más no basta conocer con claridad aquello que deseamos lograr, ni tampoco el mejor medio para poder conseguirlo. Tampoco, basta con ser capaz de; es preciso juzgarse capaz de utilizar las capacidades y las habilidades personales ante circunstancias muy diversas. La percepción de las personas acerca de su propia eficacia se alza como un requisito fundamental para desarrollar con éxito las acciones conducentes al logro de los objetivos personales. Dicha autopercepción, denominada autoeficacia, ejerce una profunda influencia en la elección de tareas y actividades, en el esfuerzo y en la perseverancia de las personas cuando se enfrentan a determinados retos e incluso en las reacciones emocionales que experimentan ante situaciones difíciles (León-Rubio et al., 2011; Pérez et al., 2011; Wolters, 
2004). En definitiva, las creencias de autoeficacia representan un mecanismo cognitivo que media entre el conocimiento y la acción y que determina, junto con otras variables, el éxito de las propias acciones (Castañeda et al., 2010; Pérez et al., 2011; Sansinenea et al., 2008; Zimmerman y Kitsantas, 2005).

Por todo ello, se puede concluir que la autoeficacia tiene un papel vital en el ámbito académico, de ahí que un buen desempeño académico no puede ser garantizado solo por los conocimientos y habilidad de los individuos. Las creencias de eficacia pueden determinar un desempeño diferente en dos personas con el mismo grado de habilidad. Esto se debe a que el éxito académico demanda procesos reguladores como la autoevaluación, el automonitoreo y el uso de estrategias metacognitivas de aprendizaje, procesos que son influidos positivamente por un alto grado de creencia en la propia capacidad o autoeficacia (Wolters, 2004). Así mismo, la autoeficacia académica representa un factor de vital importancia si se quiere formar personas que aprendan de por vida; ya que las creencias en las propias capacidades para manejar actividades académicas afectan el nivel de aspiración de los estudiantes, su preparación para diferentes carreras, además de su nivel de interés de logros intelectuales y sus éxitos académicos (Bandura, 1982, 1997). Por otro lado, en relación con la motivación académica, las creencias de autoeficacia afectan el nivel de esfuerzo, persistencia y la elección de actividades. Alumnos con un elevado sentido de eficacia para cumplir tareas educativas persistirán más ante dificultades, trabajarán con más intensidad y participarán más que aquellos que duden de sus capacidades (Bandura, 1997).

En este trabajo se analiza la consistencia interna y la estructura factorial de un instrumento de autoreporte que permite identificar conductas académicas en el ámbito sociocultural, cuyo nivel de autoeficacia percibido por los alumnos representen un área de oportunidad o mejora; en relación con el resto de los alumnos, aportando evidencias y datos que propicien la intervención educativa dentro de una perspectiva de atención a la diversidad en el aula. En el presente estudio interesa pues, no solo la estructura factorial del instrumento, sino también la equivalencia psicométrica del mismo en distintos grupos; ya que en el contexto de la comparación intergrupal, es indispensable plantearse la necesidad de llevar a cabo la adaptación de un instrumento de medida psicológica que cumpla con todos los criterios de equivalencia, pero sobre todo plantearse si la misma estructura factorial es aplicable a distintos grupos de sujetos 0 , de modo más genérico, a distintas poblaciones (Abalo et al., 2006).

\section{METODOLOGÍA}

\section{Sujetos}

La muestra de 592 sujetos, $143(24.2 \%)$ mujeres y $449(75.8 \%)$ hombres se obtuvo mediante un muestreo por conveniencia, tratando de abarcar la representatividad de las diferentes licenciaturas de Ingeniería que se ofrecen en la Universidad Autónoma de Chihuahua; luego, la muestra, fue aleatoriamente dividida en dos submuestras $(50 \%$ y $50 \%)$ utilizando el Statistical Package for the Social Sciences (SPSS) en su versión 17.0; con el fin de realizar estudios paralelos que permitieran corroborar y verificar los resultados obtenidos (validación cruzada).

La primera mitad (submuestra 1) quedo constituida por 288 sujetos; 61 (21.2\%) mujeres y 227 $(78.8 \%)$ hombres. Las edades fluctúan entre los 17 y 22 años, con una media de 18.62 y una desviación estándar de 1.26 años. La segunda mitad (submuestra 2) quedo compuesta por 304 sujetos; $82(27.0 \%)$ mujeres y $222(73.0 \%)$ hombres. Las edades fluctúan entre los 17 y 22 años, con una media de 18.46 y una desviación estándar de 1.10 años.

\section{Instrumento}

Previo a la elaboración del instrumento, cada uno de los principales dominios incluidos en la Competencia Básica Sociocultural (la cual se refiere al respeto hacia valores, costumbres, pensamientos y opiniones de los demás, apreciando y conservando el entorno) de los currículos de las licenciaturas de la Universidad Autónoma de Chihuahua, se redactaron en forma tal que los sujetos pudieran valorar la propia capacidad e interés en los mismos en una escala tipo Likert. 
Cada uno de los ítems, obtenidos tuvieron que ser corregidos de tal manera que los sujetos pudieran contestarlos en tres escenarios distintos: capacidad percibida, interés en ser capaz y de cambio en ser capaz de.

La escala de autoeficacia percibida en el ámbito sociocultural diseñada, es una encuesta tipo Likert, asistida por computadora de 9 ítems donde el encuestado responde, en una escala de 0 a 10 , que tan capaz se siente, que tanto interés tiene y si se esfuerza en cambiar que tan capaz sería en cada uno de los dominios de las competencias básicas antes mencionadas (Blanco, Martínez, Zueck y Gastélum, 2011). Se eligió este tipo de encuesta por ser fácil de construir y de aplicar; además, de proporcionar una buena base para una primera ordenación de los individuos en la característica que se mide; en nuestro caso autoeficacia (Bandura, 2006; Nunnally y Bernstein, 1995; Raviolo, Ramírez, López y Aguilar 2010).

\section{Procedimiento}

Se invitó a participar en el estudio a los alumnos de primer ingreso de las licenciaturas de Ingeniería que se ofrecen en la Universidad Autónoma de Chihuahua; los que aceptaron participar firmaron la carta de aceptación correspondiente. Luego se aplicó el instrumento antes descrito por medio de una computadora personal y utilizando el módulo administrador del instrumento del editor de escalas versión 2.0 (Blanco et al., 2007), en una sesión de aproximadamente 20 minutos; en los laboratorios o centros de cómputo de las unidades académicas participantes. Al inicio de cada sesión se hizo una pequeña introducción sobre la importancia de la investigación y de cómo acceder al instrumento. Las instrucciones de cómo responder se encontraban en las primeras pantallas; antes del primer reactivo del instrumento. Al término de la sesión se les agradeció su participación. Una vez aplicado el instrumento se procedió a recopilar los resultados por medio del módulo generador de resultados del editor de escalas versión 2.0 (Blanco et al., 2007).

\section{Análisis de datos}

El análisis psicométrico se realizó en dos etapas: 1) análisis factorial exploratorio y 2) análisis factorial confirmatorio y de invarianza factorial; con el fin de obtener una prueba que presente las mejores propiedades para la conformación de los puntajes de autoeficacia percibida en el ámbito sociocultural en universitarios de Ingeniería.

Para determinar el número mínimo de factores comunes capaces de reproducir, de un modo satisfactorio, las correlaciones observadas entre los ítems del instrumento, se realizaron sendos análisis factoriales exploratorios con las submuestras 1 y 2, a partir del método de factores principales, tomando como base el criterio de Kaiser-Guttman (Costello y Osborne, 2005), además de que para garantizar una adecuada representación de las variables (ítems), solo se conservaron aquéllos cuya comunalidad, o proporción de su varianza explicada por la solución factorial, fuera superior a .45; después de una rotación varimax (Costello y Osborne, 2005). Luego mediante el coeficiente a de cronbach se estimó la consistencia interna para cada factor retenido como una medida de su fiabilidad (Nunnally y Bernstein, 1995; Elosua y Zumbo, 2008).

Para conducir el análisis factorial confirmatorio en la submuestra 2, se utilizó el software AMOS 16 (Arbuckle, 2007), las varianzas de los términos de error fueron especificados como parámetros libres, en cada variable latente (factor) se fijó uno de los coeficientes estructurales asociados a uno, para que su escala sea igual a la de una de las variables superficiales (ítems). El método de estimación empleado fue el de Máxima Verosimilitud; siguiendo la recomendación de Thompson (2004), en el sentido de que cuando se emplea análisis factorial confirmatorio se debe corroborar no sólo el ajuste de un modelo teórico sino que es recomendable comparar los índices de ajuste de varios modelos alternativos para seleccionar el mejor.

Para evaluar el ajuste del modelo se emplearon el estadístico Chi-cuadrado, el índice de bondad de ajuste (GFI), el residuo cuadrático medio (RMR), el error cuadrático medio de aproximación (RMSEA) y el índice de validación cruzada esperada (ECVI) como medidas absolutas de ajuste. El 
índice de bondad ajustado (AGFI), el Índice Tucker-Lewis (TLI), el Índice del ajuste normal (NFI) y el índice de bondad de ajuste comparativo (CFI) como medidas de ajuste incremental. El índice de ajuste normado de parsimonia (PNFI), el índice de calidad de ajuste de parsimonia (PGFI), la razón de Chi-cuadrado sobre los grados de libertad (CMIN/GL) y el Criterio de Información de Akaike (AIC) como medidas de ajuste de parsimonia (Gelabert et al., 2011; Leighton et al., 2007).

Para comprobar la estructura factorial de la escala autoeficacia en conductas académicas obtenida a partir de la submuestra 2 (análisis factorial confirmatorio, primera solución factorial), se realizó un segundo análisis factorial confirmatorio en la submuestra 1.

\section{RESULTADOS}

\section{Análisis factorial exploratorio}

Para determinar el número mínimo de factores comunes capaces de reproducir, de un modo satisfactorio, las correlaciones observadas entre los 9 ítems del instrumento empleado, se realizó, en la submuestra 1, la extracción de éstos a partir del método de componentes principales. La significación del Test de Barlett (1238.920; p<.0001) y el KMO (.888) mostraron una adecuada correlación entre los ítems y una buena adecuación muestral respectivamente. Detectándose, después de una rotación varimax, una estructura de dos factores; el conjunto de los factores seleccionados explicaron el $64.20 \%$ de la varianza (Tablas 1 y 2 ).

Con el fin validar la solución factorial obtenida con la submuestra 1 , se realizó nuevamente un análisis de factores principales con los datos de la submuestra 2; encontrándose de nuevo una estructura de dos factores. El conjunto de los dos factores explicaron el $62.06 \%$ de la varianza (Tablas 1 y 2). La significación del Test de Barlett (1187.046; $p<.0001)$ y el KMO (.883) muestran nuevamente, una adecuada correlación entre los ítems y una buena adecuación muestral respectivamente.

Tabla 1: Ítems agrupados por factor. Análisis factorial exploratorio submuestras 1 y 2 . Soluciones rotadas.

\begin{tabular}{|c|c|c|c|c|}
\hline \multirow{2}{*}{ Ítem } & \multicolumn{2}{|c|}{ Submuestra 1} & \multicolumn{2}{|c|}{ Submuestra 2} \\
\hline & 1 & 2 & 1 & 2 \\
\hline $\begin{array}{l}6 \text { Generar una interacción con el medio ambiente, } \\
\text { impulsando el nivel cultural comunitario. }\end{array}$ & .82 & & .79 & \\
\hline $\begin{array}{l}1 \text { Participar activamente en procesos de creación, } \\
\text { conservación y difusión cultural. }\end{array}$ & .81 & & .80 & \\
\hline 9 Actuar como promotor de la calidad de vida. & .79 & & .78 & \\
\hline $\begin{array}{l}8 \text { Interactuar con diferentes grupos sociales promoviendo } \\
\text { la calidad de vida. }\end{array}$ & .78 & & .76 & \\
\hline $\begin{array}{l}7 \text { Participar en propuestas que contribuyan al desarrollo, y } \\
\text { mejoramiento social y cultural. }\end{array}$ & .73 & & .76 & \\
\hline $\begin{array}{l}5 \text { Analizar los fenómenos de globalización y desarrollo } \\
\text { sustentable a partir de diferentes perspectivas. }\end{array}$ & .71 & & .74 & \\
\hline $\begin{array}{l}3 \text { Demostrar valores ante las diferentes costumbres y } \\
\text { diferencias y hacia lo multicultural. }\end{array}$ & & .80 & & .76 \\
\hline 4 Identificarme con la cultura de mi estado y país. & & .73 & & .43 \\
\hline 2 Actuar con respeto y tolerancia & & .67 & & .83 \\
\hline
\end{tabular}


Tabla 2: Autovalores y porcentaje de varianza explicada por los factores retenidos. Análisis factorial exploratorio submuestras 1 y 2 . Soluciones rotadas.

\begin{tabular}{|l|c|c|c|c|c|c|}
\hline & \multicolumn{3}{|c|}{ Submuestra 1 } & \multicolumn{3}{c|}{ Submuestra 2 } \\
\hline \multicolumn{1}{|c|}{ Factor } & Autovalor \% varianza $\%$ acumulado & Autovalor\% varianza $\%$ acumulado \\
\hline 1. Promoción de la Cultura & 3.77 & 41.94 & 41.94 & 3.75 & 41.64 & 41.64 \\
\hline 2. Identidad Cultural & 2.00 & 22.26 & 64.20 & 1.84 & 20.42 & 62.06 \\
\hline
\end{tabular}

Fiabilidad de las subescalas (consistencia interna)

Las subescalas (factores) resultantes en los análisis factoriales exploratorios, de ambas submuestras, poseen alfas superiores a .7, evidenciando una consistencia interna adecuada para este tipo de subescalas, particularmente si se considera el número reducido de ítems (Tabla 3).

Tabla 3: Coeficientes de consistencia interna de los factores obtenidos en los análisis factoriales exploratorios submuestras 1 y 2 .

\begin{tabular}{|c|c|c|}
\hline Factor & $\alpha$ Submuestra 1 & $\alpha$ Submuestra 2 \\
\hline 1. Promoción de la Cultura & .896 & .890 \\
\hline 2. Identidad Cultural & .726 & .721 \\
\hline
\end{tabular}

\section{Análisis factorial confirmatorio}

Ahora, mediante el análisis factorial confirmatorio sobre los datos de la submuestra 2, se contrasta la solución factorial resultante del análisis factorial exploratorio realizado con los datos de la submuestra 1; con el fin de obtener modelos congenéricos y, al mismo tiempo, probar la validez de constructo de las dimensiones y variables estudiadas. De acuerdo a los resultados de la Tabla 4 el análisis factorial confirmatorio de 9 ítems agrupados en dos factores en la submuestra 2 es aceptable (GFI .928 y RMSEA .099) y de acuerdo a las medidas de ajuste incremental y de parsimonia (Tablas 5 y 6 ) significativamente superior al modelo independiente y muy similar al modelo saturado. Para comprobar la estructura factorial de la escala autoeficacia percibida en el ámbito sociocultural obtenida a partir de la submuestra 2 (análisis factorial confirmatorio, primera solución factorial), se realizó un segundo análisis factorial confirmatorio en la submuestra 1. La estructura factorial analizada plantea un modelo de dos factores, con adscripciones de los ítems basados en análisis factorial confirmatorio de la submuestra 2 (2 factores). De acuerdo a los resultados de la Tabla 4, el segundo análisis factorial confirmatorio (submuestra 1) indica que el modelo de medición de dos factores es aceptable (GFI .923 y RMSEA .105) y de acuerdo a las medidas de ajuste incremental y de parsimonia (ver Tablas 5 y 6) significativamente superior al modelo independiente y muy similar al modelo saturado.

Tabla 4: Medidas absolutas de ajuste para los modelos generados.

Análisis factorial confirmatorio submuestras 1 y $2 ;{ }^{*} p<.01$.

\begin{tabular}{|l|c|c|c|c|c|}
\hline & \multicolumn{5}{|c|}{ Índice de Ajuste } \\
\hline \multicolumn{7}{|c|}{ Modelo } & $\chi^{2}$ & GFI & RMR & RMSEA & ECVI \\
\hline \multicolumn{7}{|c|}{ Primera solución factorial (submuestra 2) } \\
\hline Independiente & $1202.977^{*}$ & .389 & 1.536 & .327 & 4.027 \\
\hline Saturado & 0 & 1 & 0 & & 0.397 \\
\hline 2 factores & $102.977^{*}$ & .928 & 0.199 & .099 & 0.465 \\
\hline \multicolumn{7}{|c|}{ Segunda solución factorial (submuestra 1) } \\
\hline Independiente & $1255.691^{*}$ & .355 & 1.608 & .344 & 4.438 \\
\hline Saturado & 0 & 1 & 0 & & 0.314 \\
\hline 2 factores & $109.017^{*}$ & .923 & 0.172 & .105 & 0.512 \\
\hline
\end{tabular}


Tabla 5: Medidas de ajuste incremental para los modelos generados.

Análisis factorial confirmatorio submuestras 1 y 2 .

\begin{tabular}{|c|c|c|c|c|}
\hline & \multicolumn{4}{|c|}{ Índice de Ajuste } \\
\hline Modelo & AGFI & TLI & NFI & CFI \\
\hline \multicolumn{5}{|c|}{ Primera solución factorial (submuestra 2) } \\
\hline Independiente & .236 & 0 & 0 & 0 \\
\hline Saturado & & & 1 & 1 \\
\hline 2 factores & .875 & .909 & .914 & .934 \\
\hline \multicolumn{5}{|c|}{ Segunda solución factorial (submuestra 1) } \\
\hline Independiente & .194 & 0 & 0 & 0 \\
\hline Saturado & & & 1 & 1 \\
\hline 2 factores & 867 & .906 & .913 & .932 \\
\hline
\end{tabular}

Tabla 6: Medidas de ajuste de parsimonia para los modelos generados.

Análisis factorial confirmatorio submuestras 1 y 2 .

\begin{tabular}{|c|c|c|c|c|}
\hline & \multicolumn{4}{|c|}{ Índice de Ajuste } \\
\hline Modelo & PNFI & PGFI & CMIN/GL & AIC \\
\hline \multicolumn{5}{|c|}{ Primera solución factorial (submuestra 2) } \\
\hline Independiente & 0 & .311 & 33.396 & 140.977 \\
\hline Saturado & 0 & & & 90.000 \\
\hline 2 factores & .660 & .536 & 3.961 & 140.977 \\
\hline \multicolumn{5}{|c|}{ Segunda solución factorial (submuestra 1) } \\
\hline Independiente & 0 & .284 & 34.880 & 1273.691 \\
\hline Saturado & 0 & & & 90.000 \\
\hline 2 factores & .660 & .533 & 4.193 & 147.017 \\
\hline
\end{tabular}

En la figura 1 se presenta el modelo de medición del primer análisis factorial confirmatorio (Submuestra 2) para los 9 ítems agrupados en dos factores, incluyendo los coeficientes de regresión estandarizados entre ítems y factores y las saturaciones factoriales estandarizadas (comunalidades) de cada uno de los ítems.

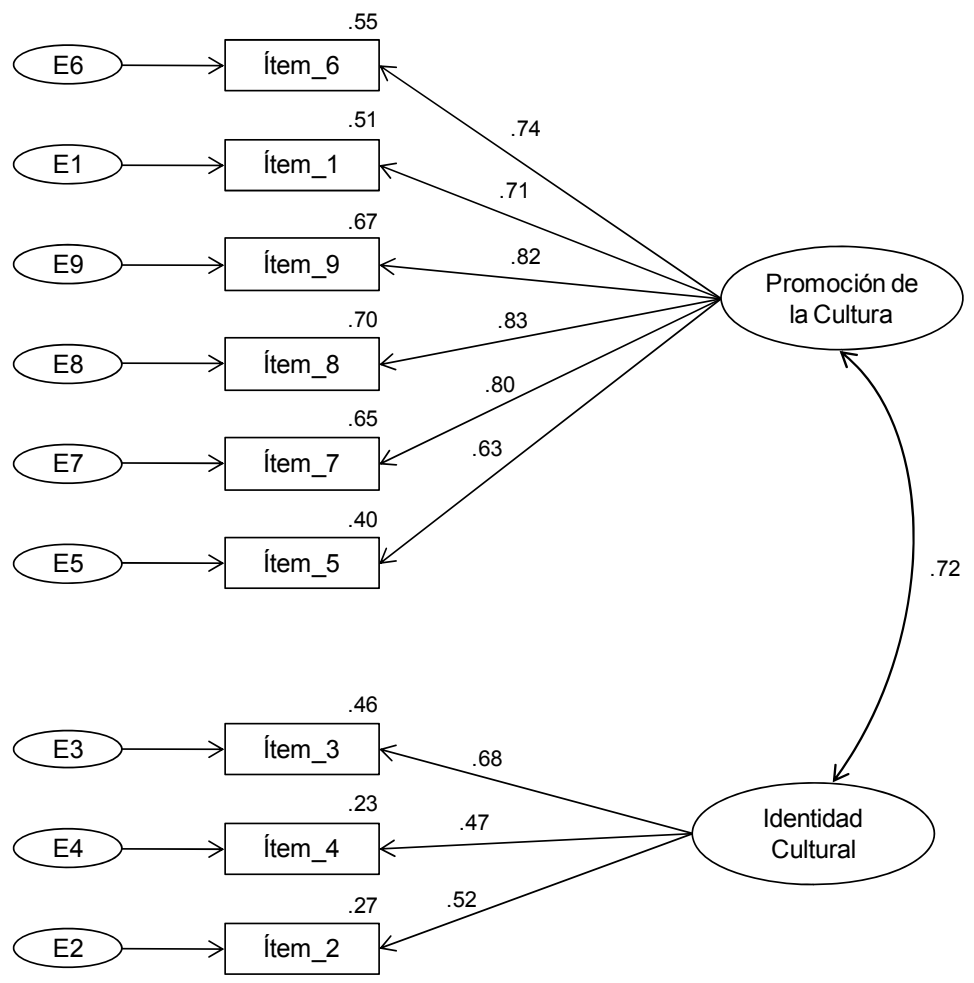

Fig. 1: Modelo de medición para la escala. Análisis factorial confirmatorio submuestra 2. Fuente: de los autores. 
En el factor Promoción de la Cultura, todos los ítems resultan bien explicados a partir del constructo hipotetizado, con saturaciones, por encima o iguales a .40. En el factor Identidad Cultural, presenta en general, saturaciones factoriales estandarizadas moderas (menores de .40), salvo en el caso del ítem 3 (Que tan capaz me siento para: Demostrar valores ante las diferentes costumbres y diferencias y hacia lo multicultural), con una saturación aceptable de .46.

Por su parte, los factores correlacionan entre sí de forma estadísticamente significativa $(p<.01)$. La correlación entre los factores Promoción de la Cultura e Identidad Cultural es alta; lo cual muestra que a medida que aumenta la autoeficacia percibida en alguno de los factores, también aumenta en el otro. En la figura 2 se presenta el modelo de medición del segundo análisis factorial confirmatorio (submuestra 1) para los 9 ítems agrupados en dos factores, incluyendo los coeficientes de regresión estandarizados entre ítems y factores y las saturaciones factoriales estandarizadas (comunalidades) de cada uno de los ítems.

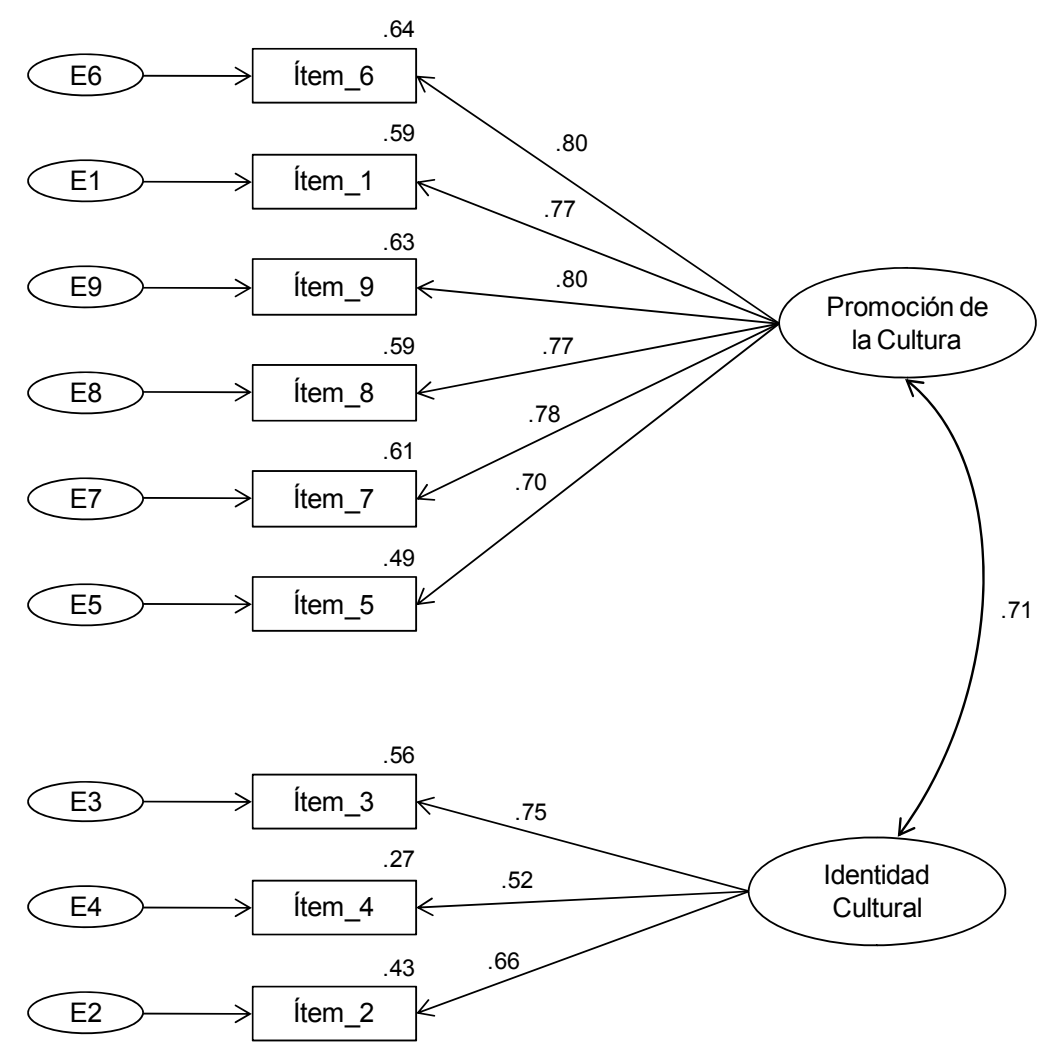

Fig. 2: Modelo de medición para la escala. Análisis factorial confirmatorio submuestra 1. Fuente: de los autores.

De nuevo y de manera similar a los resultados obtenidos en la solución factorial con la submuestra 2, en el factor Promoción de la Cultura, todos los ítems resultan bien explicados a partir del constructo hipotetizado, con saturaciones, iguales o por encima de .45. El factor Identidad Cultural, presenta en general, saturaciones factoriales estandarizadas elevadas (mayores a .40), salvo en el caso del ítem 4 (Que tan capaz me siento para: Identificarme con la cultura de mi estado y país), con una saturación moderada de .27. Por su parte, los factores correlacionan entre sí de forma estadísticamente significativa $(p<.01)$. La correlación entre los factores Promoción de la Cultura e Identidad Cultural es alta; lo cual muestra que a medida que aumenta la autoeficacia percibida en alguno de los factores, también aumenta en el otro.

Para analizar la invarianza factorial del cuestionario se siguieron las recomendaciones de Abalo et al. (2006) estimando el mismo modelo en ambas submuestras; los índices de ajuste obtenidos (Tabla 7) permiten aceptar la equivalencia de los modelos de medida básicos entre las dos submuestras. Aunque el valor de Chi-cuadrado excede al exigido para aceptar la hipótesis de invarianza, el resto de índices contradicen esta conclusión (GFI .926; CFI .933; RMSEA .072; AIC 287.995) lo que nos permite aceptar el modelo base de la invarianza (modelo sin restricciones). 
Añadiendo al modelo base restricciones sobre las cargas factoriales caracterizamos la invarianza métrica. Los valores que se recogen en la tabla 7 permiten aceptar este nivel de invarianza. El índice de ajuste general (GFI= .918) y el error cuadrático medio de aproximación (RMSEA $=.071)$ siguen aportando información convergente en esta dirección. Además, el criterio de información de Akaike $(A I C=295.187)$ y el índice comparativo de Bentler $(C F I=.927)$ no sufren grandes variaciones respecto al modelo anterior. Haciendo uso del criterio para la evaluación de los modelos anidados propuesto por Cheung y Rensvold (2002), quiénes sugieren que si el cálculo de la diferencia de los CFI de ambos modelos anidados disminuye en .01 o menos, se da por bueno el modelo restringido y por tanto el cumplimiento de la invarianza factorial; la diferencia entre CFIs obtenida permite aceptar el modelo de invarianza métrica. Podemos concluir hasta ahora que las cargas factoriales son equivalentes en las dos muestras.

Una vez demostrada la invarianza métrica entre las muestras, pasamos a evaluar la equivalencia entre interceptos (invarianza factorial fuerte). Los índices (Tabla 7) muestran un buen ajuste de este modelo, tanto evaluado de modo independiente como analizándolo respecto a su anidamiento con el modelo de invarianza métrica. La diferencia entre los índices comparativos de Bentler es menor que .001; el índice de ajuste general es .917 y el error cuadrático medio de aproximación es .069. Aceptada la invarianza fuerte, los dos modelos evaluados son equivalentes respecto a los coeficientes factoriales y a los interceptos.

Tabla 7 Índices de bondad de ajuste de cada uno de los modelos puestos a prueba en la invarianza factorial; ${ }^{*} p<.01$.

\begin{tabular}{|l|l|c|c|c|c|c|c|}
\hline \multicolumn{1}{|c|}{ Modelo } & \multicolumn{7}{|c|}{ Índice de Ajuste } \\
\hline & $\chi^{2}$ & gl & GFI & NFI & CFI & RMSEA & AIC \\
\hline Modelo sin restricciones & $211.991^{*}$ & 52 & .926 & .914 & .933 & .072 & 287.995 \\
\hline Invarianza métrica & $233.187^{*}$ & 59 & .918 & .905 & .927 & .071 & 295.187 \\
\hline Invarianza factorial fuerte & $235.919^{*}$ & 62 & .917 & .904 & .927 & .069 & 291.919 \\
\hline
\end{tabular}

\section{CONCLUSIONES}

El Análisis factorial exploratorio del instrumento autoeficacia en el ámbito sociocultural llevado a cabo en cada una de las submuestras estudiadas puso de manifiesto una estructura factorial de dos componentes: Promoción de la Cultura e Identidad Cultural. Los componentes de ambas submuestras evidenciaron una consistencia interna óptima, particularmente si se considera el número reducido de ítems en cada uno de ellos. Por otra parte, el Análisis Factorial Confirmatorio indicó que el ajuste de los datos al modelo teórico de 9 ítems agrupados en dos factores es adecuado. Al mismo tiempo que los dos factores así obtenidos presentan en general saturaciones factoriales estandarizadas adecuadas. Por su parte los dos factores correlacionan entre sí de forma positiva y estadísticamente significativa lo cual muestra que a medida que aumenta la autoeficacia percibida en alguno de los factores, también aumenta en el otro. Conjuntamente con todo lo antes dicho, los resultados del análisis de la invarianza factorial entre las submuestras; indican una alta congruencia entre pares de componentes. Lo que sugiere la existencia de fuertes evidencias de la validación cruzada de la medida y por tanto de la estabilidad de la estructura, hasta que no se demuestre lo contrario.

En síntesis, el análisis de las propiedades psicométricas, ha mostrado que una estructura bifactorial es viable y adecuada de acuerdo a los requisitos psicométricos establecidos cuando los informantes son los propios alumnos. La estructura de dos factores, atendiendo a criterios estadísticos y sustantivos, ha mostrado adecuados indicadores de ajuste, de fiabilidad y de validez. Sin embargo, y como ha sido indicado por diferentes investigadores, la validez factorial de los instrumentos de medida debe ser demostrada con muestras que presenten diferencias tanto poblacionales como culturales, de tal manera que, consideramos que más estudios son necesarios con el fin de corroborar o refutar los datos obtenidos en la presente investigación. 
Por último, tomando en cuenta que la investigación empírica ha demostrado, de manera amplia, que la autoeficacia resulta ser más predictiva del rendimiento académico que otras variables cognitivas (Bandura, 1982), que logra predecir el éxito posterior (Bandura, 1997; Ornelas et al., en prensa) y que es un importante mediador cognitivo de competencia y rendimiento (Vera et al., 2011) en cuanto favorece los procesos cognoscitivos (Carbonero y Merino, 2008; Ornelas et al., 2011) concluimos que darse a la tarea de mejorar la percepción de ser capaz en quien aprende es un objetivo educacional valioso, bajo el supuesto implícito de que su potenciación servirá como vehículo para la mejora de otros resultados tales como el logro académico y la autoestima. Tomando en cuenta que la continua conciencia de fracaso reduce las expectativas de éxito y no favorece en ningún modo ni el aprendizaje ni el desarrollo personal.

\section{REFERENCIAS}

Abalo, J., Lévy, J., Rial, A., y Varela, J., Invarianza factorial con muestras múltiples. En J. Lévy (Ed.), Modelización con Estructuras de Covarianzas en Ciencias Sociales pp. 259-278. Netbiblo, Madrid (2006).

Adeyemo, D., Moderating Influence of Emotional Intelligence on the Link Between Academic Selfefficacy and Achievement of University Students, Psychology Developing Societies, 19(2), 199-213 (2007).

Arbuckle, J., AMOS users guide version 16.0 (2007).

Bandura, A., Self-efficacy mechanism inhuman agency", American Psychologist, 37( 2), 122-147 (1982).

Bandura, A., Self-efficacy: The exercise of Control, Freeman, New York (1997).

Bandura, A. Guide for constructing self-efficacy scales. En F. Pajares y T. Urdan (Eds.), Selfefficacy beliefs of adolescents, pp. 307-337. CT: Information Age Publishing, Greenwich (2006).

Blanco, A., Creencias de autoeficacia de estudiantes universitarios: un estudio empírico sobre la especificidad del constructo, RELIEVE, 16(1), 1-28 (2010).

Blanco, H., Martínez, M., Zueck, M. A. y Gastélum, G., Análisis psicométrico de la escala autoeficacia en conductas académicas en universitarios de primer ingreso, Actualidades Investigativas en Educación, 11(3), 1-27 (2011).

Blanco, H. y otros siete autores, Editor para la construcción y aplicación de escalas por medio de una PC, Tecnociencia Chihuahua, 1(1), 55-59 (2007). http://tecnociencia.uach.mx/numeros/v1n1/ data/Editor_para_la_Construccion_y_Aplicacion_de_Escalas_por_medio_de_una_PC.pdf

Brown, S., y otros cinco autores, Social cognitive predictors of college students' academic performance and persistence: a meta-analytic path analysis, Journal of Vocational Behavior, 72(3), 298-308 (2008).

Caprara, G. y otros siete autores, Longitudinal analysis of the role of perceived efficacy for selfregulated learning in academic continuance and achievement, Journal of Educational Psychology, 100, 525-534 (2008).

Carbonero, M. A. y Merino, E., Autoeficacia y madurez vocacional, Psicothema, ISSN: 1886-144X (en línea), 16(2), 229-234, 2008. http://www.psicothema.com/pdf/1187.pdf. Acceso: 3 de agosto (2011).

Cartagena, M., Relación entre la autoeficacia y el rendimiento escolar y los hábitos de estudio en alumnos de secundaria, Revista Iberoamericana sobre Calidad, Eficacia y Cambio en Educación,. 6(3), 59-99 (2008). 
Castañeda, S., Pineda, M. L., Gutierrez, E., Romero, N., Peñalosa, E., Construcción de instrumentos de estrategias de estudio, autorregulación y epistemología personal. Validación de constructo, Revista Mexicana de Psicología, 27(1), 77-85 (2010).

Cheung, G. W. y Rensvold, R. B., Evaluating goodness-of-fit indexes for testing measurement invariance, Structural Equation Modeling, 9, 233-255 (2002).

Costello, A. y Osborne, J., Best practices in exploratory factor analysis: four recommendations for getting the most from your analysis. Practical Assessment, Research and Evaluation, ISSN: 15317714, 10(7), 1-9, 2005. http://pareonline.net/pdf/v10n7.pdf. Acceso: 9 de noviembre (2011)

Elosua, P. y Zumbo, B., Coeficientes de fiabilidad para escalas de respuesta categórica ordenadas, Psicothema, ISSN: 1886-144X (en línea), 20(4), 896-901, 2008. http://www.psicothema.com/pdf/3572.pdf. Acceso: 3 de diciembre (2011).

Gelabert, E. y otros cinco autores, Psychometric properties of the Spanish version of the Frost Multidimensional Perfectionism Scale in women, Psicothema, ISSN: 1886-144X (en línea), 23(1), 133-139, 2011. http://www.psicothema.com/pdf/3861.pdf. Acceso: 3 de noviembre (2011).

Leighton, J., Gokiert, R. y Cui, Y., Using Exploratory and Confirmatory Methods to Identify the Cognitive Dimensions in a Large-Scale Science Assessment, International Journal of Testing, 7(2), 141-189 (2007).

León-Rubio, J. M., Cantero, F. J. y León-Pérez, J. M., Diferencias del rol desempeñado por la autoeficacia en el burnout percibido por el personal universitario en función de las condiciones de trabajo, Anales de Psicología, 27(2), 518-526. (2011).

Nunnally, J. y Bernstein, I., Teoría Psicométrica, McGraw-Hill, México (1995).

Ornelas, M., Blanco, H., Gastélum, G. y Chávez, A., Autoeficacia percibida en conductas académicas en mujeres universitarias, Form. Univ., 5(2), en prensa (2012).

Ornelas, M., Blanco, H., Rodríguez, J. M. y Flores, F. J., Análisis Psicométrico de la Escala Autoeficacia en Conductas de Cuidado de la Salud Física en Universitarios de Primer Ingreso, Form. Univ., 4(6), (2011).

Pérez, E., Lescano, C., Zalazar, P., Furlám, L. y Martínez, M., Desarrollo y análisis psicométricos de un Inventario de Autoeficacia para Inteligencias Múltiples en Niños Argentinos. Psicoperspectivas, ISSN: 0718-6924 (en línea), 10 (1), 169-189, 2011. http://www.psicoperspectivas.cl/index.php/psicoperspectivas/article/viewFile/141/129. Acceso: 3 de diciembre (2011).

Raviolo, A., Ramirez, P., López, E. A. y Aguilar, A., Concepciones sobre el Conocimiento y los Modelos Científicos: Un Estudio Preliminar, Form. Univ., ISSN: 0718-5006 (en línea), 3(5), 29-36, 2010. http://www.scielo.cl/pdf/formuniv/v3n5/art05.pdf. Acceso: 3 de diciembre (2011).

Salanova, M.; Llorens, S. y Shaufeli, W. B., "Yes, I can, I feel good, and I just do it!" On gain cycles and spirals of efficacy beliefs, affect, and engagement, Applied Psychology: An International Review, 60, 255-285 (2011).

Sansinenea, E. y otros seis autores, Autoconcordancia y autoeficacia en los objetivos personales: ¿Cuál es su aportación al bienestar?, Anales de Psicología, 24(1), 121-128 (2008).

Thompson, B., Exploratory and Confirmatory Factor Analysis. Understanding concepts and applications, American Psychological Association, Washington, D C: (2004). 
Vera, M., Salanova, M. y Martín-del-Río, B., Self-efficacy among university faculty: how to develop an adjusted scale, Anales de Psicología, 27(3), 800-807 (2011).

Wolters, C., Advancing achievement goal theory: using goal structures and goal orientations to predict students motivation, cognition and achievement, Journal of Educational Psychology, 96(2), 236-250 (2004).

Zimmerman, B. y Kitsantas, A., Homework practice and academic achievement. The mediating role of self-efficacy and perceived responsibility beliefs, Contemporary Educational Psychology, 30(4) 397-417 (2005). 